Canadian Journal of Physics

Canadian

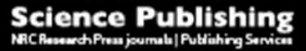

Revue canadienne de physique

\title{
An efficient quantum teleportation of six-qubit state via an eight-qubit cluster state
}

\begin{tabular}{|r|l|}
\hline Journal: & Canadian Journal of Physics \\
\hline Manuscript ID & cjp-2017-0527.R1 \\
\hline Manuscript Type: & Article \\
\hline Complete List of Authors: & $\begin{array}{l}\text { Zhao, Nan; Xidian University } \\
\text { Li, Min; Xidian University } \\
\text { Chen, Nan; Xidian University } \\
\text { Pei, Xing; Xidian University }\end{array}$ \\
\hline Keyword: & $\begin{array}{l}\text { Quantum teleportation, Cluster state, Eight-qubit state, Deterministic } \\
\text { scheme, Higher efficiency }\end{array}$ \\
\hline $\begin{array}{r}\text { Is the invited manuscript for } \\
\text { consideration in a Special } \\
\text { Issue? : }\end{array}$ & N/A \\
\hline &
\end{tabular}




\title{
An efficient quantum teleportation of six-qubit state via an eight-qubit cluster state
}

\author{
Nan Zhao*, Min $\mathrm{Li}^{*}$, Nan Chen*, Chang-xing Pei*
}

\begin{abstract}
We present a scheme for teleporting a certain class of six-partical state via an eight-partical cluster state as quantum channel. In our scheme, the sender merely needs to perform an eight-partical von-Neumann projective measurement, and the receiver gives a corresponding general evolution to restore the original state. Our scheme is a deterministic scheme. Compared with other schemes proposed before, our scheme possesses higher intrinsic efficiency.
\end{abstract}

\section{INTRODUCTION}

Quantum teleportation is an important research content in quantum information, and it has the main research value in quantum computation and quantum communication. By combining with classical communication and quantum entanglement resources, quantum teleportation realizes the remote transmission of unknown quantum states. With the in-depth study of quantum teleportation in theory and experiment, scientific researchers have made a lot of achievements. In 1993, quantum teleportation was proposed firstly by Bennett et al. [1]. In 1997, J. W. Pan and Bobst were firstly realized the remote transmission of unknown quantum states [2]. In 2009, University of Science and Technology of China and Tsinghua University completed $16 \mathrm{~km}$ free space quantum teleportation between Badaling Great Wall and Huailai County [3]. In 2012, J.W. Pan et al. realized the hundred kilometers of free space quantum teleportation and entanglement distribution [4]. In 2016, F. Grosshans used city dark fiber to achieve 3.7 miles (about 6km) quantum state transmission[5]. In addition, high dimensional entanglement and hyperentanglement are attracting much attention recently [6], [7], [8], [9], [10], [11], [12]. In 2011, Y.B. Sheng proposed teleportation of multi-degree of freedom [6], and it is demonstrated in the experiment [7]. Preparation of high-dimensional entanglement has made a breakthrough [8], [9], [10], [11]. The mathematics of teleportation involves the anti-conjugate operation, which is thoroughly reviewed in [12]. In 2017, Z.Y. Zhou presented a quantum twisted double-slit experiment to address the question that whether quantum states are real or not [13]. Moreover, teleportation is crucial in distributed quantum learning [14].

Since the concept of quantum teleportation is proposed, researches about theory of quantum teleportation, the experimental analysis [2], [3], [4] and the application of assumptions have never ceased. Recently, quantum teleportation becomes a hot spot in quantum information research field, and researchers

\footnotetext{
${ }^{1}$ State Key Laboratory of Integrated Services Networks, Xidian University, $\mathrm{Xi}^{\prime}$ an, 710071, China

Corresponding author: Nan Zhao (email: zhaonan@xidian.edu.cn).
}

have proposed some novel schemes using different entangled states as quantum channel such as EPR state [5], W-like state [15], [16], GHZ state [17], [18], [19], [20], GHZ-like [21], [22], [23] state, Cluster state et al. Cluster state [24] is a new multi-particle entangled state proposed by H. J. Briegel and R. Raussendorf in 2001. When the number of particles satisfies $N>3$, cluster state presents their unique characteristics, namely maximum connectedness and a high persistency of entanglement. Recently, a number of quantum teleportation schemes have been proposed by utilizing cluster states as a quantum channel. X. W. Wang et al. [25] presented scheme for quantum teleportation of an arbitrary two-particle state by using one-dimensional cluster state. Transferring a threeparticle state by utilizing a five-particle cluster state of QT is proposed by Zhou and Liu [26]. Binayak S. Choudhury proposed a quantum teleportation protocol of three-qubit State utilizing four-qubit quantum channels [27].

Research about the preparation of cluster state has been constantly in progress, and it has made a breakthrough in theory and experiment. A number of schemes of preparing cluster state have been proposed, such as photons [28], ions [29], atoms [30] and cavity fields [31]. Preparations of a twoparticle cluster state and a four-particle cluster state were proposed respectively [32], [33]. Preparing an eight-particle entangled state in cavity QED is proposed by Li, Sang and Nie [34]. In terms of experiment, the four-particle cluster state and the six-particle cluster state were generated under laboratory conditions [35], [36]. In Ref.[37], Nie et al. proposed a quantum information splitting scheme with controlled-NOT operations to teleport an arbitrary three-qubit GHZ-type state. All above contents motivate us to study the applications of six-qubit and eight-qubit cluster states.

In this paper, we utilize an eight-qubit cluster state to teleport a six-qubit state. Firstly, the sender Alice operates an eight-particle von-Neumann projective measurement. Then, Alice informs the receiver Bob of her measurement results by the classical channel. Finally, Bob gives a corresponding general evolution, and restores the target states. The successful probability of our scheme is 1 .

\section{MAIN RESULT}

\section{A. Quantum Teleportation of six-qubit state}

Alice wants to transmit an unknown six-qubit entangled state to a distant receiver Bob. The six-qubit pure quantum state is described as

$$
\begin{aligned}
& |\iota\rangle_{a b c d e f}=(\alpha|000000\rangle+\beta|000010\rangle \\
& +\gamma|111101\rangle+\delta|111111\rangle)_{\text {abcdef }}
\end{aligned}
$$


where $|\alpha|^{2}+|\beta|^{2}+|\gamma|^{2}+|\delta|^{2}=1$. Alice wants to teleport the state $|\chi\rangle_{\text {abcdef }}$ to Bob.

Suppose one quantum channel shared between Alice and Bob : an eight-qubit cluster state is given by,

$$
\begin{aligned}
& |\vartheta\rangle_{12345678}=(\alpha|00000000\rangle+\beta|00001001\rangle \\
& +\gamma|11110110\rangle+\delta|11111111\rangle)_{12345678}
\end{aligned}
$$

where the qubits a, b, c, d, e, f, 7 and 8 belong to Alice, qubits $1,2,3,4,5$ and 6 belong to Bob, respectively.

Therefore, the state of the whole system composed of an unknown six-qubit state and a quantum channel is given by

$$
|\kappa\rangle=|\iota\rangle_{a b c d e f} \otimes|\vartheta\rangle_{12345678}
$$

The details of the state of the whole system is listed in Equation 21.

The mutually orthonormal eight-qubit states in Alice's possession $\left|\theta^{i}\right\rangle_{\text {adcdef78 }}(i=1,2, \cdots, 16)$ are given by,

$$
\begin{aligned}
& \left|\theta^{1}\right\rangle_{\text {abcdef78 }}=\frac{1}{2}(\alpha|00000000\rangle+\beta|00001001\rangle \\
& +\gamma|11110110\rangle+\delta|11111111\rangle)_{a b c d e f 78} \\
& \left|\theta^{2}\right\rangle_{\text {abcdef78 }}=\frac{1}{2}(\alpha|00000000\rangle-\beta|00001001\rangle \\
& +\gamma|11110110\rangle-\delta|11111111\rangle)_{a b c d e f 78} \\
& \left|\theta^{3}\right\rangle_{\text {abcdef78 }}=\frac{1}{2}(\alpha|00000000\rangle+\beta|00001001\rangle \\
& -\gamma|11110110\rangle-\delta|11111111\rangle)_{a b c d e f 78} \\
& \left|\theta^{4}\right\rangle_{\text {abcdef78 }}=\frac{1}{2}(\alpha|00000000\rangle-\beta|00001001\rangle \\
& -\gamma|11110110\rangle+\delta|11111111\rangle)_{a b c d e f 78} \\
& \left|\theta^{5}\right\rangle_{\text {abcdef78 }}=\frac{1}{2}(\alpha|00000001\rangle+\beta|00001000\rangle \\
& +\gamma|11110111\rangle+\delta|11111110\rangle)_{a b c d e f 78} \\
& \left|\theta^{6}\right\rangle_{\text {abcdef78 }}=\frac{1}{2}(\alpha|00000001\rangle-\beta|00001000\rangle \\
& +\gamma|11110111\rangle-\delta|11111110\rangle)_{a b c d e f 78} \\
& \left|\theta^{7}\right\rangle_{\text {abcdef78 }}=\frac{1}{2}(\alpha|00000001\rangle+\beta|00001000\rangle \\
& -\gamma|11110111\rangle-\delta|11111110\rangle)_{a b c d e f 78} \\
& \left|\theta^{8}\right\rangle_{\text {abcdef78 }}=\frac{1}{2}(\alpha|00000001\rangle-\beta|00001000\rangle \\
& -\gamma|11110111\rangle+\delta|11111110\rangle)_{a b c d e f 78} \\
& \left|\theta^{9}\right\rangle_{\text {abcdef78 }}=\frac{1}{2}(\alpha|00000010\rangle+\beta|00001000\rangle \\
& +\gamma|11110111\rangle+\delta|11111101\rangle)_{a b c d e f 78} \\
& \left|\theta^{10}\right\rangle_{\text {abcdef78 }}=\frac{1}{2}(\alpha|00000010\rangle-\beta|00001000\rangle \\
& +\gamma|11110111\rangle-\delta|11111101\rangle)_{a b c d e f 78}
\end{aligned}
$$

$$
\begin{aligned}
& \left|\theta^{11}\right\rangle_{a b c d e f 78}=\frac{1}{2}(\alpha|00000010\rangle+\beta|00001000\rangle \\
& -\gamma|11110111\rangle-\delta|11111101\rangle)_{a b c d e f 78} \\
& \left|\theta^{12}\right\rangle_{a b c d e f 78}=\frac{1}{2}(\alpha|00000010\rangle-\beta|00001000\rangle \\
& -\gamma|11110111\rangle+\delta|11111101\rangle)_{a b c d e f 78} \\
& \left|\theta^{13}\right\rangle_{\text {abcdef78 }}=\frac{1}{2}(\alpha|00000011\rangle+\beta|00001010\rangle \\
& +\gamma|11110101\rangle+\delta|11111110\rangle)_{a b c d e f 78} \\
& \left|\theta^{14}\right\rangle_{\text {abcdef78 }}=\frac{1}{2}(\alpha|00000011\rangle-\beta|00001010\rangle \\
& +\gamma|11110101\rangle-\delta|11111100\rangle)_{a b c d e f 78} \\
& \left|\theta^{15}\right\rangle_{a b c d e f 78}=\frac{1}{2}(\alpha|00000011\rangle+\beta|00001010\rangle \\
& -\gamma|11110101\rangle-\delta|11111100\rangle)_{a b c d e f 78} \\
& \left|\theta^{16}\right\rangle_{a b c d e f 78}=\frac{1}{2}(\alpha|0000011\rangle-\beta|00001010\rangle \\
& -\gamma|11110101\rangle+\delta|11111100\rangle)_{a b c d e f 78}
\end{aligned}
$$

In our scheme, firstly, Alice operates an eight-qubit vonNeumann projective measurement on particles a, b, c, d, e, f, 7, 8. Then, Alice transmits the measurement result to Bob during the classical channel. Bob operates a corresponding unitary transformation on his particles. Finally, the state of $\mathrm{Bob}^{\prime}$ s particle is changed to the target state. Our scheme is a deterministic scheme and the successful probability is 1 . The measurement results and the unitary transformation are listed in Table 1.

\section{B. Comparisons with other schemes}

To illustrate the superiority of our scheme intuitively, we make some comparisons between our scheme and other typical schemes presented before in references [27], [38]. Firstly we give some concise reviews on the $\mathrm{Z}$ and $\mathrm{B}$ schemes.

In [27], Binayak S. Choudhury and Arpan Dhara presented a perfect teleportation protocol for certain class of three-qubit entangled states. They use three four-qubit cluster states as quantum channels. Firstly, Alice performs three four-qubit von-Neumann projective measurements on particles (a, 1, 2, 3), (b, 5, 6, 7), and (c, 9 10, 11), respectively. Then, Alice sends her measurement results to Bob, Bob applies the appropriate unitary operations on his qubits 4,8 , and 12 . Finally, the target state is restored.

In [38], Zhu H.P. proposed a scheme for quantum teleportation of an arbitrary two-qubit state via GHZ-like states. He use two GHZ-like states as quantum channels. Its basic idea can be described as that Alice performs two three-qubit vonNeumann projectivemeasurements, Bob can restore the target state by operating appropriate unitary transformations. 


$$
\begin{aligned}
& |\kappa\rangle=|\iota\rangle_{a b c d e f} \otimes|\vartheta\rangle_{12345678} \\
& =\frac{1}{4}\left[\left|\theta^{1}\right\rangle_{\text {abcdef } 78}(\alpha|000000\rangle+\beta|000010\rangle+\gamma|111101\rangle+\delta|111111\rangle)_{123456}\right. \\
& +\left|\theta^{2}\right\rangle_{\text {abcdef78 }}(\alpha|000000\rangle-\beta|000010\rangle+\gamma|111101\rangle-\delta|111111\rangle)_{123456} \\
& +\left|\theta^{3}\right\rangle_{\text {abcdef78 }}(\alpha|000000\rangle+\beta|000010\rangle-\gamma|111101\rangle-\delta|111111\rangle)_{123456} \\
& +\left|\theta^{4}\right\rangle_{\text {abcdef78 }}(\alpha|000000\rangle-\beta|000010\rangle-\gamma|111101\rangle+\delta|111111\rangle)_{123456} \\
& +\left|\theta^{5}\right\rangle_{\text {abcdef78 }}(\alpha|000010\rangle+\beta|000000\rangle+\gamma|111111\rangle+\delta|111101\rangle)_{123456} \\
& +\left|\theta^{6}\right\rangle_{\text {abcdef78 }}(\alpha|000010\rangle-\beta|000000\rangle+\gamma|111111\rangle-\delta|111101\rangle)_{123456} \\
& +\left|\theta^{7}\right\rangle_{\text {abcdef78 }}(\alpha|000010\rangle+\beta|000000\rangle-\gamma|111111\rangle-\delta|111101\rangle)_{123456} \\
& +\left|\theta^{8}\right\rangle_{\text {abcdef78 }}(\alpha|000010\rangle-\beta|000000\rangle-\gamma|111111\rangle+\delta|111101\rangle)_{123456} \\
& +\left|\theta^{9}\right\rangle_{\text {abcdef78 }}(\alpha|111101\rangle+\beta|111111\rangle+\gamma|000000\rangle+\delta|000010\rangle)_{123456} \\
& +\left|\theta^{10}\right\rangle_{\text {abcdef78 }}(\alpha|111101\rangle-\beta|111111\rangle+\gamma|000000\rangle-\delta|000010\rangle)_{123456} \\
& +\left|\theta^{11}\right\rangle_{\text {abcdef } 78}(\alpha|111101\rangle+\beta|111111\rangle-\gamma|000000\rangle-\delta|000010\rangle)_{123456} \\
& +\left|\theta^{12}\right\rangle_{\text {abcdef78 }}(\alpha|111101\rangle-\beta|111111\rangle-\gamma|000000\rangle+\delta|000010\rangle)_{123456} \\
& +\left|\theta^{13}\right\rangle_{\text {abcdef78 }}(\alpha|111111\rangle+\beta|111101\rangle+\gamma|000010\rangle+\delta|000000\rangle)_{123456} \\
& +\left|\theta^{14}\right\rangle_{\text {abcdef } 78}(\alpha|111111\rangle-\beta|111101\rangle+\gamma|000010\rangle-\delta|000000\rangle)_{123456} \\
& +\left|\theta^{15}\right\rangle_{\text {abcdef78 }}(\alpha|111111\rangle+\beta|111101\rangle-\gamma|000010\rangle-\delta|000000\rangle)_{123456} \\
& \left.+\left|\theta^{16}\right\rangle_{\text {abcdef78 }}(\alpha|111111\rangle-\beta|111101\rangle-\gamma|000010\rangle+\delta|000000\rangle)_{123456}\right]
\end{aligned}
$$

Table 1. Strategy for recovering the six-qubit state.

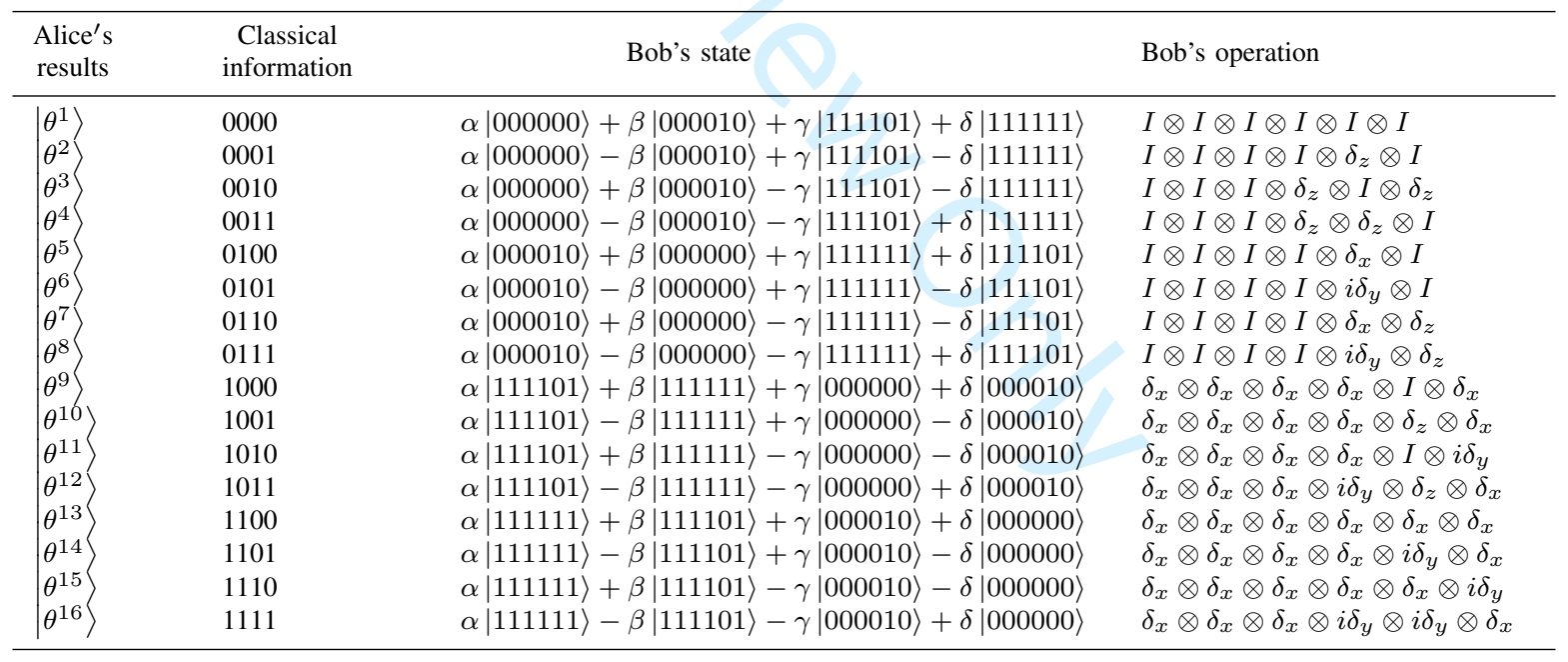

Now let us compare our scheme with the above two schemes. The intrinsic efficiency of the communication scheme is defined [39] as

$$
\eta=q_{s} /\left(q_{u}+b_{t}\right)
$$

where $q_{s}$ is the number of qubits that consist of the quantum information to be exchanged, $q_{u}$ is the number of the qubits which are used as the quantum channel (except for those chosen for security checking), and $b_{t}$ is the classical bits transmitted.

According to Equation 12, we calculate the intrinsic efficiency of our scheme and references [27], [38], the $\eta$ is $6 / 16,2 / 12$ and $3 / 24$ respectively. We can find that the $\eta$ of our scheme is higher. In addition, our scheme only needs one quantum channel, the $\mathrm{Z}$ and $\mathrm{B}$ schemes need 2 and 3 quantum channels respectively. Our scheme consumes less quantum resources.

By comparison above, our scheme has better performance. Moreover, the generations of the six-particle and eight-particle cluster state have been achieved in laboratories respectively references [35], [36]. That means that our scheme based on the eight particle cluster channel is feasible within the reach of current technologies. 


\section{CONCLUSION}

In summary, we propose a deterministic scheme for the teleportation of a six-particle state. In our scheme, Alice operates an eight particle projective measurement, and Bob can reconstruct the teleported state by the measurement results from Alice. In section 2, we also have compared our scheme with other schemes proposed recently on four aspects. Comparisons show that our scheme possesses higher intrinsic efficiency, needs fewer quantum channels and consumes less quantum resources. In addition, based on the existing technologies our scheme is feasible.

\section{ACKNOWLEDGMENT}

This work is supported by the National Natural Science Foundation of China (Grant No. 61301171 and Grant No. 61372076), the Fundamental Research Funds for the Central Universities (JB No.160115).

\section{REFERENCES}

[1] C. H. Bennett, G. Brassard, C. Crepeau, R. Jozsa, A. Peres and W. K. Wootters 1993 Phys. Rev. Lett. 70 1895-1899

[2] J. W. Pan and A. Zeilinger 1999 PHYSICS 10005

[3] X. M. Jin, J. G. Ren, B. Yang, et al. 2010 Nature Photonics 4(6) 376-381

[4] J. Yin, J. G. Ren, H. Lu, et al. 2012 Nature 488(7410) 185-188

[5] F. Grosshans 2016 Nature Photonics 10 623-625

[6] Y. B. Sheng, F. G. Deng and G. L. Long 2010 Phys. Rev. A 82(3) 032318

[7] X. L. Wang, X. D. Cai, Z. E. Su, et al.2015 Nature 518(7540) 516-519

[8] R. Heilmann, M. Grafe, et al. 2015 Science bulletin 60(1) 96-100

[9] F. G. Deng, B. C. Ren and X. H. Li 2017 Science Bulletin 62(1) 46-68

[10] T. Li and Z. Q. Yin 2016 Science Bulletin 61(2) 163-171

[11] J. Dong, Y. M. Chen, et al.2017 Science Bulletin 62(18) 1235-1238

[12] C. M. Xie, Y. M. Liu, et al. 2016 SCIENCE CHINA Physics, Mechanics \& Astronomy 59(10) 100314

[13] Z. Y. Zhou, Z. H. Zhu, et al. 2017 Science Bulletin 62 1185-1192

[14] Y. B. Sheng and L. Zhou 2017 Science Bulletin 62(14) 1025-1029

[15] G. Yang, B. W. Lian, M. Nie and J. Jiao 2017 Chin. Phys. B 26(4) 040305

[16] J. Heo and C. H. Hong, et al.2015 Chin. Phys. B 24(5) 050304

[17] Q. Y. Wen and T. Y. Wang 2011 Chin. Phys. B 20(4) 040307

[18] W. Li, X. W. Zha and J. X. Qi 2016 Int. J. Theor. Phys. 55 3927-3933

[19] F. G. Deng and C. Y. Li , et al.2005 Phys. Rev. A 72 22338-22345

[20] K. Li and F. Z. Kong, et al.2016 Quantum Inf. Process. 15 3137-3150

[21] P. Agrawal and A. Pati 2006 Phys. Rev. A 7462320

[22] M. Y. Wang and F. L. Yan 2011 Chin. Phys. B 20(12) 120309

[23] H. Shima and H. Monireh 2016 Quantum Inf. Process. $15905-912$

[24] E. Pakhshan and P. Pouria 2014 Int. J. Theor. Phys. 13 1789-1811

[25] X. W. Wang and Y. G. Shan 2007 Phys. Lett. A 364 7-11

[26] Z. M. Liu and L. Zhou 2014 Int. J. Theor. Phys. 53(12) 4079-4082

[27] S. C. Binayak, Arpan and D 2016 Int. J. Theor. Phys. 55 3393-3399

[28] D. C. Liand Z. L. Cao 2007 Commun. Theor. Phys. 47(3) 464-466

[29] W. W. Yang and Y. L. He, et al. 2007 Chin. Phys. Lett. 24(4) 104-120

[30] S. H. Xiang and K. H. Song 2006 Chin. Phys. Lett. 23(6) 1466

[31] P. P. Munhoz and F. L. SemioA 2008 Phys. Lett. A 372(12) 3580-3584

[32] H. Wunderlich and C. Wunderlich, et al. 2009 Phys. Rev. A 79052324

[33] L. M. Duan and H. J. Kimble 2004 Phys. Rev. Lett. 92(12) 127902

[34] Y. H. Li and M. H. Sang, et al. 2016 Int. J. Theor. Phys. 55(11) 46934698

[35] P. Walther and K. J. Resch, et al. 2005 Nature 434 269-276

[36] C. Y. Lu and X. Q. Zhou, et al. 2007 Nature 3(2) 91-95

[37] Y. H. Li, M. H. Sang and Y. Y. Nie 2016 Int. J. Theor. Phys. $554693-$ 4698

[38] H. P. Zhu 2014 Int. J. Theor. Phys. 53 4095-4097

[39] H. Yuan, Y. M. Liu, W. Zhang and Z. J. Zhang 2008 J. Phys. B 41 145506 